\title{
Respuesta e interpretación a políticas de rendición de cuentas de formación docente en Chile
}

\author{
Responses and Interpretation of Teacher Training \\ Accountability Policies in Chile
}

\author{
María Beatriz Fernández Cofré1, Loreto Fernández Quevedo \\ Mónica Diaz Navarro², y Patricia Jofré Cáceres ${ }^{2}$ \\ ${ }^{1} \mathrm{CIAE}$, Instituto de Estudios Avanzados en Educación, Universidad de Chile \\ ${ }^{2}$ Universidad Alberto Hurtado
}

\section{Resumen}

Diferentes países han generado políticas para controlar la formación docente mediante regulaciones y rendición de cuentas desde los años 90. Sin embargo, existe escasa y contradictoria evidencia sobre los efectos de estas políticas. En Chile, la rendición de cuentas y la desregulación operan simultáneamente en la formación docente. En 2016 se aprobó la política de Sistema de Desarrollo Profesional Docente, que incluye la acreditación obligatoria de programas, el aumento gradual de la selectividad, la renovación de los estándares, y la implementación de pruebas diagnósticas para estudiantes de pedagogía. Este estudio cualitativo exploratorio analiza, a través de entrevistas semiestructuradas, cómo 40 directores de programas de pedagogía de 10 universidades chilenas ponen en práctica - interpretan y responden a- esta política. Utilizando un análisis de contenido cualitativo, se identificaron cuatro grupos de interpretaciones y respuestas, cuyas diferencias pueden entenderse con base en dimensiones contextuales que median las concepciones de profesionalismo, justicia y relaciones de poder expresadas por los participantes. Este estudio contribuye a comprender los elementos que influyen en la puesta en práctica de políticas de formación docente, a expandir la escasa literatura empírica en esta área, y a informar a quienes toman decisiones de política y forman docentes.

Palabras claves: política gubernamental, formación inicial, investigación cualitativa.

\section{Correspondencia a:}

María Beatriz Fernández

CIAE, Instituto de Estudios Avanzados en Educación, Universidad de Chile.

Periodista José Carrasco Tapia № 75, Santiago, Chile.

mbeatriz.fernandez@uchile.cl

Este trabajo fue financiado por CONICYT/FONDECYT INICIACIÓN 11170663 y

CONICYT PCI/REDI 170506. Se agradece el financiamiento otorgado por ANID/PIA/

Fondos Basales para Centros de Excelencia FB0003.

(c) 2021 PEL, http://www.pensamientoeducativo.org - http://www.pel.cl

ISSN:0719-0409 DDI:203.262, Santiago, Chile doi: 10.7764/PEL.58.1.2021.9 


\title{
Abstract
}

\begin{abstract}
Since the 1990s, various countries have created policies aimed at controlling teacher training through regulation and accountability. However, empirical evidence on the effects of these policies is scarce and contradictory. In Chile, accountability and deregulation work in tandem in teacher training. In 2016, the Chilean government approved the policy for the Professional Development System for Teachers, including mandatory accreditation for teacher training programs, a gradual increase in selectivity for prospective teachers, renewal of standards, and implementation of diagnostic tests for student teachers. Through semi-structured interviews, this qualitative exploratory study analyzes how 40 directors of teacher training programs in 10 Chilean universities enact-interpret and respond to-this policy. Using a qualitative content analysis, we identified four groups of interpretations and responses, for which the differences could be understood based on contextual dimensions that modulate conceptions of professionalism, justice, and power relationships expressed by the participants. This study contributes to the understanding of the aspects that influence the enactment of teacher training policies, expanding the scant empirical research in this area, and informing policymakers and teacher trainers.
\end{abstract}

Keywords: Public policy, teacher education, qualitative research.

Esta investigación busca analizar cómo interpretan y responden los directores de carreras de pedagogía en Chile al reciente aumento de regulaciones y rendición de cuentas en formación docente del Sistema de Desarrollo Profesional Docente (SDPD), incremento enmarcado en una tendencia internacional. Desde los años noventa, bajo la idea de una modernización capitalista, predomina el esfuerzo por disminuir el rol del Estado, desregularizar mercados, liberar relaciones comerciales y privatizar organismos públicos en varios países. El sector educativo fue considerado clave en estas transformaciones, argumentándose que el desarrollo de competencias y conocimientos de los individuos aumentaría la productividad y posibilitaría la inserción de las economías nacionales en el nuevo escenario global (Ball, 2001). La calidad de la enseñanza se estableció como elemento esencial para la competitividad económica, señalándose a la formación docente como un elemento clave para fortalecerla (Cochran-Smith et al., 2018).

El modelo neoliberal en educación y los principios de la nueva gestión pública han orientado las transformaciones educacionales mediante la incorporación de políticas que permiten el control a distancia, utilizando estándares, acreditaciones e incentivos (Anderson \& Cohen, 2018; Verger, 2013). Estos cambios son señalados como reformas educativas de segunda vía, caracterizados por una creciente centralización y estandarización de los objetivos educativos desde los gobiernos (Hargreaves y Shirley, 2012). Asimismo, la elección, competencia e incentivos por resultados, forman parte de la agenda educativa global y la reestructuración de los sistemas educativos. Estos cambios han sido promovidos por organismos internacionales, argumentando que están presentes en países de alto rendimiento en pruebas estandarizadas internacionales (Fernández, 2018). Estas reformas se apoyan en una narrativa de desconfianza docente, caracterizada por afirmaciones normativas e interrelacionadas sobre cómo mejorar la formación docente y la docencia (Cochran-Smith et al., 2018; Zeichner, 2010).

La implementación de políticas de rendición de cuentas refleja efectos contradictorios en los lugares en los que se han implementado, sin evidencia concluyente respecto de su impacto en resultados de aprendizaje, autonomía docente y procesos institucionales (Parcerisa y Verger, 2016). Si bien la regulación y rendición de 
cuentas aumentaron en la formación docente en las últimas dos décadas, existe escasa información empírica sobre la implementación de estas políticas y sus consecuencias (Bartlett, Otis-Wilborn, \& Peters, 2017; Hasbun \& Rudolph, 2016; Ledwell \& Oyler, 2016; Peck, Gallucci, \& Sloan, 2010). Algunos autores señalan que la definición de estándares y sistemas de evaluación externa para programas de pedagogía y profesores mejorarán las capacidades docentes y el estatus de la profesión (Darling-Hammond \& Lieberman, 2012; Hickok, 1998). Sin embargo, advierten que para que se produzcan los efectos esperados se deben implementar a gran escala, utilizarlos para orientar el aprendizaje docente, y contar con un cuerpo de conocimientos, habilidades y actitudes comunes legítimos para la profesión. Para Sotomayor y Gysling (2011), las políticas basadas en estándares no son sinónimo de estandarización educativa, y promueven el mejoramiento de la calidad cuando son sostenidas por un amplio acuerdo político y parte de una agenda de acciones.

Otros investigadores plantean que las políticas de rendición de cuentas, que promueven la estandarización, devalúan la dimensión ético profesional en la formación docente (Butin, 2005). El debilitamiento de esta dimensión implica el riesgo de contar con aulas eficaces, pero injustas y antidemocráticas (Escudero, 2011). Fullan (2013) identifica la rendición de cuentas punitiva entre los impulsores incorrectos de reformas. Señala que este elemento puede estar presente, pero no debe tener un rol central en reformas a gran escala. Los impulsores correctos se centran en la construcción de capacidad, trabajo en equipo, pedagogía y políticas sistémicas. Otros autores critican que el contexto, la diversidad y la cultura han sido reducidos u omitidos por las visiones instrumentalistas de la enseñanza y el aprendizaje de estas políticas. Además, cuestionan que las nociones de equidad sostenidas a través de mecanismos de rendición de cuentas externas se limitan a buscar reducir las brechas en rendimiento académico, sin atender a temas complejos de justicia social (Fernández, 2018).

Sin embargo, diversos investigadores han advertido que las políticas educativas no son linealmente implementadas en los espacios locales, y su éxito dependerá de los conocimientos, actitudes y percepciones de los actores (Bolívar, Gallego, León, y Pérez, 2005) y cómo sean traducidas, recreadas y resignificadas (Ball, Maguire, \& Braun, 2012). La historia y situación de la formación docente en Chile hace relevante explorar cómo las políticas de rendición de cuentas se vivencian en diferentes contextos.

La aplicación del modelo neoliberal en Chile ha tenido una de las expresiones más radicales a nivel mundial, instalándose en todos los ámbitos, incluido el educativo desde los años 70. Las regulaciones introducidas en formación docente, desde mediados del año 2000, intentaron incidir en un sistema altamente desregulado y privatizado (Fernández, 2018). En la última década, estas políticas transitaron desde la regulación indirecta con consecuencias bajas e incentivos focalizados, a la regulación con altas consecuencias, mediante medidas contenidas en el SDPD, aprobado el 2016 (Fernández y Madrid, 2020). Esta política incluye la acreditación obligatoria de los programas de formación docente, el aumento progresivo de requerimientos de ingreso a estos programas, la implementación de dos pruebas diagnósticas para los estudiantes de pedagogía, y la renovación de los estándares para la formación docente $(\mathrm{BCN}, 2016)$. En esta política, existe una tensión entre la consolidación de un paradigma educativo basado en estándares, con presión por rendimiento contra incentivos, y un intento por la profesionalización y la generación de competencias profesionales (Ruffinelli, 2016).

Esto hace relevante el estudio de las interpretaciones y respuestas al SDPD de directores de programas que trabajan en universidades de diferentes características. Este estudio analiza estas interpretaciones y los cambios, desde sus perspectivas, en las decisiones curriculares y de admisión en sus programas como respuesta a la política. Los resultados de esta investigación contribuyen a comprender los elementos contextuales que influyen en la implementación de políticas de formación docente e identificar temáticas que median la interpretación y la respuesta de formadores docentes a ellas: concepciones de profesionalización, justicia social y relaciones de poder. Estos hallazgos expanden la escasa literatura empírica en esta área, y pueden ser de utilidad para quienes toman decisiones y forman docentes. 


\section{Políticas de formación docente en Chile}

La ley 20.903 (BCN, 2016) dio origen a la política del SDPD y tiene como propósito "dignificar la profesión docente, apoyar su ejercicio y aumentar su valoración" (CPEIP, 2017, p. 8), para contribuir a los aprendizajes del estudiantado. Esta política creó un sistema que regula e integra en un solo cuerpo legal la formación inicial docente (FID), el ingreso a la docencia y el desarrollo de una carrera profesional.

La política se propone responder a las problemáticas que han afectado al cuerpo docente en Chile, desde la década de los 70, como consecuencia del shock neoliberal que, entre otras medidas, desreguló la oferta de programas de formación docente. El decreto de 1981 permitió crear nuevas instituciones de educación superior y facultó a organismos no universitarios para impartir programas de pedagogía. Por lo anterior, la formación docente se trasladó ampliamente a los institutos profesionales, que absorbieron en 1985 casi la mitad de la matrícula (Elacqua, Hincapie, Vegas, y Alfonso, 2018).

Los gobiernos democráticos realizaron cambios, pero continuaron la senda neoliberal (Inzunza, Assaél, y Scherping, 2011). Desde 2008, con el propósito de monitorear la calidad y avanzar en la regulación de la formación docente, se aplicó una prueba de salida a estudiantes de pedagogía (Prueba INICIA). Sin embargo, esta iniciativa recibió críticas, porque se focalizó en el control y la rendición de cuentas (Montecinos, 2014). Su legitimidad se cuestionó porque los resultados fueron utilizados para ranquear instituciones y no eran representativos, dado el carácter voluntario de la prueba (García-Huidobro, 2011).

Según la división de la educación superior, la oferta de programas de FID creció en torno a 200\% entre los años 2000 y 2009, existiendo más de 1500 programas de formación y más de 120.000 estudiantes. Esta tendencia al alza se frenó a partir de 2013, debido a la introducción de regulaciones e incentivos como estándares y una prueba de salida (Ruffinelli, 2016).

La alta masividad de los programas de pedagogía estuvo acompañada de una baja selectividad. En el año 2015, coexistían programas de pedagogía con y sin procesos de selectividad; más de 90.000 estudiantes se distribuían en 70 instituciones de educación superior y 863 programas, no estando acreditados casi un 28\% de estos. En 2016, 47\% de los estudiantes ingresaron a universidades privadas sin rendir la Prueba de Selección Universitaria (PSU). Los estudiantes que rindieron la PSU alcanzaron en promedio un puntaje cercano al percentil 50 (Elacqua et al., 2018).

Frente a este panorama, bajo un intenso debate político, en 2016 se aprueba el SDPD, política que regula la oferta y el aseguramiento de la formación docente en las universidades. Para resolver la desregulación de la oferta, la política establece que los programas de pedagogía deben estar acreditados para recibir nuevos estudiantes. Además, define como única institución acreditadora la Comisión Nacional de Acreditación (Ruffinelli, 2016). La política regula también los criterios de selectividad de las universidades e instala como obligatoria la rendición de la PSU, junto con la obtención de puntajes mínimos en esta evaluación, o la ubicación dentro de un percentil determinado en el ranking de egreso de la enseńanza secundaria. Estas exigencias se aplican de forma gradual en los procesos de selección, aumentando cada tres años desde 2017 hasta 2023. Como vía alternativa de ingreso, se permiten cupos para estudiantes de programas de acceso inclusivo, impartidos por las universidades y reconocidos por el Ministerio de Educación.

Además, la política regula la calidad de estudiantes de pedagogía mediante la aplicación de dos evaluaciones diagnósticas censales, una al inicio de la formación, a cargo de las universidades, y otra un año antes de finalizar el proceso (en reemplazo de la Prueba INICIA), que aplica el Ministerio de Educación, guiada por los estándares pedagógicos y disciplinarios definidos por esta institución (BCN, 2016). Esta medida busca responsabilizar a las instituciones formadoras de docentes, obligándolas a implementar planes remediales con sus estudiantes según los resultados de la segunda evaluación (Ruffinelli, 2016). 


\section{Marco teórico}

Las perspectivas tradicionales de análisis de políticas se focalizan en su planificación, implementación o evaluación, asumiendo relaciones lineales (Diem, Young, Welton, Cumings Mansfield, \& Lee, 2014; Stone, 2012). Desde la perspectiva crítica sociológica, en la que se basa este estudio, las políticas se comprenden como el resultado de procesos complejos de negociación y sujetas a cambios en los contextos locales (Ball, 1993). Las políticas educativas pueden estudiarse como un ciclo en que se identifican tres contextos (Bowe, Ball, \& Gold, 1992): 1) contexto de influencia: los discursos y grupos que afectan su construcción; 2) contexto de producción del texto: el discurso oficial de la política y el contexto en que se crea, y 3) contexto de práctica: el contexto local donde la política es interpretada, traducida y cambiada.

La puesta en práctica de políticas (policy enactment) en los contextos locales o de práctica involucra la interpretación y traducción de las políticas de manera activa por los sujetos locales (Ball et al., 2012). La interpretación se asocia al proceso de decodificar o hacer sentido a una política, mientras que la traducción se asocia al proceso de recodificación o conversión de la política en prácticas, conceptos, materiales y procedimientos en el contexto local (Ball, Maguire, Braun, \& Hoskins, 2011). Los tomadores de decisiones de políticas no pueden controlar los significados y efectos de las políticas en los contextos locales. Las personas y organizaciones tienen historias, intereses y valores que median la interpretación y la respuesta a las políticas (Ball, 1993; Ball et al., 2012). Los conocimientos, actitudes y percepciones de los actores influyen en la puesta en práctica y el éxito de las reformas (Bolívar et al., 2005). Cuatro dimensiones contextuales influyen en la puesta en práctica de políticas en las escuelas: cultura profesional (valores y creencias), contexto externo (incentivos o presiones externas), contexto situado (características del lugar y el perfil de estudiantes), y contexto material (recursos disponibles) (Ball et al., 2012).

La mayoría de los estudios empíricos que utilizan la perspectiva de puesta en práctica de políticas se centran en el sistema escolar. A nivel internacional, los estudios empíricos que analizan el proceso de implementación de una reforma, política o evaluación en la formación de profesores a nivel nacional, estatal o local examinan las experiencias y respuestas de formadores docentes y estudiantes de pedagogía a: 1) evaluaciones para los estudiantes de pedagogía (Bartlett et al., 2017; Ledwell \& Oyler, 2016; Peck et al., 2010); 2) orientaciones, evaluaciones o acreditaciones de programas de pedagogía (Hasbun \& Rudolph, 2016; Petrelli, 2014), o 3) reformas nacionales más amplias, que incluyen cambios en la formación docente (Mitchell \& Romero, 2010).

Los estudios que se centran en procesos de evaluación o acreditación de programas de pedagogía señalan los desafíos de estos procesos, y mencionan sugerencias como contar con un equipo que lidere el proceso, la creación de una cultura de rendición de cuentas y relaciones de mutuo beneficio con establecimientos escolares (Hasbun \& Rudolph, 2016). Los estudios también mencionan tensiones reportadas por formadores docentes respecto de la implementación de evaluaciones externas a estudiantes de pedagogía, debido a la escasa consideración de aspectos contextuales y la baja participación de formadores docentes en el proceso de definición de los estándares e instrumentos de evaluación (Bartlett et al., 2017; Reagan, Schram, McCurdy, Chang, \& Evans, 2016). Sin embargo, en los estudios empíricos no se reportan las interpretaciones, respuestas y experiencias de los formadores docentes ante políticas tan complejas como el SDPD, que combina estándares y evaluaciones para los estudiantes de pedagogía, exigencias de acreditación de programas de pedagogía, y el aumento de los requisitos de admisión.

Este estudio combina la perspectiva de puesta en práctica de políticas —interpretación y traducción en contextos locales - con un modelo de análisis de políticas de formación docente (Cochran-Smith et al., 2018) que examina los fundamentos de la política (valores, propósitos y conceptos), los problemas y soluciones propuestos por la política, y las relaciones de poder (mecanismos de control, contenido de la rendición de cuentas y consecuencias para la docencia y el trabajo de los formadores docentes). Estos elementos guiaron las decisiones metodológicas. 


\section{Metodología}

Esta investigación es parte de un estudio mayor, que busca comprender las respuestas e interpretaciones de formadores de profesores al SDPD. En este artículo se reportan los hallazgos de un estudio cualitativo exploratorio que, basado en un paradigma interpretativo (Creswell, 2009), se centra en cómo 40 jefes de carrera de pedagogía interpretan y responden al SDPD desde su propia perspectiva a través de entrevistas semiestructuradas. La muestra de participantes se seleccionó mediante un muestreo intencional (Wood y Smith, 2018), usando dos criterios: cargo e institución de trabajo. Se seleccionaron directores de programas de pedagogía, quienes son encargados formalmente de conectar las políticas nacionales e institucionales con el cuerpo docente, y ocupan una posición relevante en las decisiones curriculares de sus programas. Se seleccionó a directores de programas en universidades que contaran con carreras de educación de párvulos, básica, media en lenguaje y media en matemática simultáneamente, y que representaran la diversidad de universidades chilenas considerando siete aspectos: ubicación geográfica (BCN, 2018); tipos de administración y régimen de propiedad (públicas, privadas miembros del Consejo de Rectores de Universidades Chilenas, o instituciones privadas); selectividad, puntajes de ingreso PSU previos a los requerimientos del SDPD (Torres y Zenteno, 2011); calidad, años de acreditación de la universidad (Muñoz y Blanco, 2013); adscripción a la gratuidad (MINEDUC, 2018); orientación (religiosa o laica), y tamaño de matrícula de la universidad (Torres y Zenteno, 2011).

La muestra corresponde a 40 directores de programas de pedagogía (10 de educación básica, 10 de educación parvularia, 10 de educación media en lenguaje y 10 de educación media en matemáticas) ubicados en 10 universidades diferentes.

Tabla 1

Muestra

\begin{tabular}{|c|c|c|c|c|c|c|}
\hline \multirow{2}{*}{$\begin{array}{l}\text { Número } \\
\text { entrevistados }\end{array}$} & \multirow{2}{*}{$\begin{array}{cc}\text { Cargo } \\
s \quad \text { (director programa) }\end{array}$} & \multicolumn{5}{|c|}{ Características lugar de trabajo } \\
\hline & & Zona & Administración & Selectividad & Acreditación & Tamaño \\
\hline 4 & $\begin{array}{c}\text {-Básica } \\
\text {-Párvulos } \\
\text {-Media Lenguaje (ML) } \\
\text {-Media Matemáticas (MM) }\end{array}$ & Sur & $\begin{array}{l}\text { Privada } \\
\text { CRUCH }\end{array}$ & Baja & Media & Menor \\
\hline 4 & $\begin{array}{l}\text {-Básica } \\
\text {-Párvulos } \\
\text {-ML } \\
\text {-MM }\end{array}$ & Centro & Privada & Alta & Moderada & Mayor \\
\hline 4 & $\begin{array}{l}\text {-Básica } \\
\text {-Párvulos } \\
\text {-ML } \\
\text {-MM }\end{array}$ & Centro & $\begin{array}{l}\text { Privada } \\
\text { CRUCH }\end{array}$ & Alta & Alta & Mayor \\
\hline 4 & $\begin{array}{l}\text {-Básica } \\
\text {-Párvulos } \\
\text {-ML } \\
\text {-MM }\end{array}$ & Norte & Pública & Alta & Media & Menor \\
\hline 4 & $\begin{array}{l}\text {-Básica } \\
\text {-Párvulos } \\
\text {-ML } \\
\text {-MM }\end{array}$ & Sur & Pública & Alta & Media & Menor \\
\hline
\end{tabular}




\begin{tabular}{|c|c|c|c|c|c|c|}
\hline 4 & $\begin{array}{c}\text {-Básica } \\
\text {-Párvulos } \\
\text {-ML } \\
\text {-MM }\end{array}$ & Centro & Privada & Alta & Alta & Menor \\
\hline 4 & $\begin{array}{c}\text {-Básica } \\
\text {-Párvulos } \\
\text {-ML } \\
\text {-MM }\end{array}$ & Norte & Pública & Alta & Media & Menor \\
\hline 4 & $\begin{array}{c}\text {-Básica } \\
\text {-Párvulos } \\
\text {-ML } \\
\text {-MM }\end{array}$ & Centro & $\begin{array}{l}\text { Privada } \\
\text { CRUCH }\end{array}$ & Alta & Alta & Mayor \\
\hline 4 & $\begin{array}{c}\text {-Básica } \\
\text {-Párvulos } \\
\text {-ML } \\
\text {-MM }\end{array}$ & Centro & Privada & Baja & Media & Mayor \\
\hline 4 & $\begin{array}{c}\text {-Básica } \\
\text {-Párvulos } \\
\text {-ML } \\
\text {-MM }\end{array}$ & Centro & Privada & $\begin{array}{c}\text { Sin } \\
\text { Información }\end{array}$ & Moderada & Menor \\
\hline
\end{tabular}

Fuente: elaboración propia.

Las entrevistas con los participantes tuvieron un promedio de 90 minutos, con un protocolo semiestructurado basado en la perspectiva teórica, sometido a juicio de dos expertos y piloteado. El protocolo se centró en las características de los programas y los cambios para la formación docente contenidos en la política del SDPD. Las entrevistas se analizaron mediante un análisis de contenido (Cáceres, 2003), usando el software Atlas-ti. Se utilizó un árbol de códigos para identificar los fundamentos, construcción de problemas y soluciones, y las relaciones de poder e implicancias de políticas de formación docente y su puesta en práctica (Cochran-Smith et al., 2018), y se identificaron códigos emergentes basados en la información de las entrevistas. Cerca de 15\% de las entrevistas fueron doble codificadas con un índice de acuerdo sobre 75\% (Campbell, Quincy, Osserman, \& Pedersen, 2013).

\section{Resultados}

Identificamos cuatro grupos de discursos predominantes en las interpretaciones y respuestas a la política de directores de programas de pedagogía.

\section{Responsabilización y alineamiento}

En este grupo, categorizamos las respuestas de 12 directores de programas que trabajan en las tres universidades (Universidades 3, 6 y 8) con mayores años de acreditación en la muestra y administración privada, pertenecientes y no al CRUCH. Estos directores interpretan que la política se orienta a solucionar la desregulación y baja responsabilización institucional en la formación de profesores, y el bajo estatus de la profesión docente. Desde su perspectiva, la política define a los profesores como profesionales capaces de responder a la diversidad de los estudiantes y sus contextos, y a las demandas de la sociedad actual. Esto incluye la responsabilización de los profesores por el aprendizaje de sus estudiantes y su desarrollo profesional. De acuerdo con ellos, la política 
conceptualiza a los programas de pedagogía como unidades que deben asumir responsabilidad por su calidad, respondiendo positivamente al aumento de requerimientos y alineando sus currículums a los estándares nacionales para estudiantes de pedagogía. Esto se ejemplifica en el siguiente extracto:

P: ... Esa visión de la docencia... es que este profesor sea más profesional, sea investigador y tenga este rol más destacado dentro de la sociedad, está igual más teñido de este rol al que se le puede decir lo que tiene que hacer, no como en otras carreras (Director Pedagogía Básica, U3).

P: La formación inicial docente fue regida por una economía de mercado... se desregularizó la educación. Cada uno de estos elementos... ha venido [a] regular y dar una base mínima común que nos permita cumplir ciertos estándares de calidad, que es lo que se está exigiendo... [Lo que] no puedes transar es una formación de calidad para poder responder a las necesidades que tienen las personas, los nińos y las nińas y los jóvenes que están asistiendo en el sistema escolar... Es responsabilizarme como país, de la realidad que tenemos en este minuto (Director Educación Parvularia, U8).

Esta construcción discursiva del problema de la formación de profesores y la docencia es similar a la que se establece explícitamente en los documentos de la política. Además, mencionan que en general sus prácticas están alineadas con las orientaciones de política, y que tienen injerencia en estas decisiones, como se manifiesta a continuación:

P: ... La evaluación del penúltimo año, por lo que nos han contado los estudiantes, ... es súper parecida al examen de licenciatura que nosotros hacemos... En general nosotros...hemos sido provisores, y en general toda nuestra normativa siempre ha estado acorde a la ley, y cuando se han hecho procesos de desarrollo, se nos ha invitado a participar en la elaboración de estos aspectos, entonces siempre ha habido concordancia... Entonces no hemos necesitado hacer cambios (Director Educación Parvularia, U8).

Así, interpretan que la propuesta de cambio de la política es coherente con su cultura profesional (Ball et al., 2012), lo que podría relacionarse con su valoración positiva sobre sus estrategias y propósitos. Los participantes de este grupo mencionan que no han desarrollado cambios relevantes en sus procesos institucionales, currículums y procesos de admisión como respuesta a la política nacional. Antes de la aprobación de la política, sus universidades implementaron procesos internos de evaluación y rendición de cuentas en todos sus programas de pedagogía. Esta información se ha utilizado para mejorar y articular las prácticas (enfatizadas en la política nacional) y el área de didácticas. De esta manera, los recursos (contexto material) previos de la institución para hacer estos cambios les permitieron estar preparados para las exigencias de la política (Ball et al., 2012).

La respuesta de estos jefes de carrera también puede relacionarse con el contexto externo o presiones externas percibidas (Ball et al., 2012). Sus programas eran altamente selectivos antes de la aprobación de la política, por lo que no reportaron cambios en el perfil de ingreso de sus estudiantes, y sus universidades contaban con los más altos niveles de acreditación. Por lo tanto, es entendible que el incremento de selectividad y la acreditación obligatoria no fueran interpretadas como una amenaza o desafío, y que no se sintieran presionados a desarrollar cambios curriculares.

\section{Críticas a la lógica de mercado, pero compromiso con el llamado público}

Ocho de los directores de programas de pedagogía de dos universidades públicas regionales (Universidad 4 y 7 ), con acreditación media, interpretan la política nacional como un control externo de requerimientos mínimos basado en un enfoque técnico y en la desconfianza hacia la formación docente. Asocian el control externo con un enfoque gerencial y una perspectiva de educación de mercado, que contrasta fuertemente con su perspectiva pública, sus valores humanistas y su concepción de justicia. En sus programas existían procesos iniciales para evaluar los resultados de los estudiantes y asegurar la calidad de sus egresados, pero la información había sido utilizada de forma desigual entre los programas. 
Estos directores interpretan que la política asume que un buen profesor es aquel que está alineado con los estándares nacionales y las regulaciones externas. Además, señalan que la política considera que un buen profesor está conectado con la realidad de su sala de clases y se adapta a la sociedad, reproduciendo el orden social. Esto contrasta con su compromiso con el bien público y la construcción de una sociedad con valores humanistas. Como describen algunos de los participantes:

P: ...Con la irrupción fuerte en el mercado en el quehacer social... se ve a la educación como una entidad también mesurable, medible, como si fuera cualquier otra, donde hay conducta de entrada, y hay un proceso, y hay una salida, y hay instancias que están debidamente reguladas objetivadas porque en el fondo tu funcionas como una empresa, donde todos tiene que tener evidencia, todos tienen que dar cuenta de ciertos estándares, todos tienen que dar cuenta de ciertos dineros y todo se regula... De repente yo lo hallo hasta mercantilista.... Nosotros tenemos muy claro el rol que juega las instituciones [públicas] como la nuestra, y por lo tanto proyectamos el ejercicio de la labor docente siempre pensando en el beneficio de la comunidad... en la cual estamos insertos (Director Pedagogía en Lenguaje, U7).

P: El fin último de formar personas con un desarrollo de la interculturalidad más focalizado tiene que ver con construir una sociedad más respetuosa, una sociedad más equitativa, una sociedad cuyos principios sean la complementariedad más que la competencia.... Si lográsemos eso, avanzaríamos hacia una sociedad distinta respecto de la que tenemos... la educación... determina la sociedad que tenemos, cuando en la educación dejamos que se mercantilice... llegamos a la sociedad en la que estamos hoy día (Director Educación Parvularia, U4).

La cultura profesional de los participantes (Ball et al., 2012), sus creencias y valores, son considerados como contradictorios a la lógica, valores y concepciones que promueve la política. Destacan su énfasis en formar a estudiantes de pedagogía con un compromiso ético, y capaces de responder a la diversidad de los estudiantes y sus contextos, educando desde una perspectiva holística, considerando aspectos actitudinales y culturales. Esta discrepancia es también evidente al mencionar el compromiso con sus estudiantes, su contexto situado (Ball et al., 2012). Los directores mencionan que atienden a estudiantes que ingresan con bajas competencias básicas (lectura, escritura y matemáticas) y escasos hábitos de estudio, mencionando su alto compromiso con la equidad al atender a este grupo. Plantean la importancia de ofrecer apoyo académico y socioemocional en la formación docente, y la necesidad de mejorar sus cursos didácticos y disciplinares en sus programas. Los directores de programas de este grupo también critican el reduccionismo de la PSU como mecanismo de ingreso para estudiantes de pedagogía, debido a que omite aspectos actitudinales. En este grupo, dos programas no pudieron matricular estudiantes el año 2018 a raíz del aumento de requerimientos de selectividad o acreditación.

A pesar de estas críticas, estos directores mencionan que han implementado cambios en su currículum en respuesta a la política, con la finalidad de alinearse con los estándares para estudiantes de pedagogía vigentes, como se ejemplifica a continuación:

P: ... Como consecuencia de esto una actualización de las asignaturas... Nosotros teníamos por ejemplo un perfil de egreso genérico, muy tradicional, de redacción media novelesca, versus un perfil de egreso con estándares que tiene una composición absolutamente distinta... Dentro de lo genérico está el libre albedrio, o está también está libertad académica, pedagógica, de abordar; en cambio ahora, con los estándares, la cosa es distinta, está absolutamente dirigido, está absolutamente marcado lo que hay que hacer, definido, entonces no hay lugar a esa libre cátedra, sino que tiene que estar mucho más centrado en lo que está declarado (Director Pedagogía Básica, U7). 
No obstante, valoran que estas modificaciones hayan sido desarrolladas mediante procesos democráticos que, a su juicio, es la forma regular en la que se toman decisiones e implementan cambios en sus programas. Sin embargo, expresaron su preocupación por la falta de recursos para hacerse cargo de los requerimientos de la política. A diferencia del primer grupo, los recursos (contexto material) con que cuentan sus carreras influyeron negativamente en las interpretaciones de los requerimientos de la política (Ball et al., 2012).

\section{Tensión entre la política y el sello del programa}

Al igual que en el segundo grupo, los 12 directores de programas de tres universidades (1, 5 y 10) critican la política, al considerarla un control externo sobre la formación docente. Estos programas están ubicados en universidades regionales lejanas a la capital, con baja o media acreditación. Señalan que están comprometidos con atender a estudiantes de sus comunidades, que normalmente alcanzan puntajes bajos en la PSU, e ingresan con bajas competencias básicas y hábitos de estudio. Los directores de este grupo destacan su compromiso con ofrecer una formación relevante para su contexto, con énfasis local, regional o religioso. En contraste, plantean que la política nacional enfatiza la estandarización, definiendo un buen profesor como uno alineado con los estándares nacionales para estudiantes de pedagogía, conceptualizando a los profesores como ejecutores de políticas externas. Así, presentan tensiones entre su cultura profesional (Ball et al., 2012) y la política, como el grupo anterior.

Estos participantes critican, también, fuertemente el aumento de selectividad de la política. Indican que esta prueba no evalúa aspectos actitudinales, como la vocación o el compromiso con la enseńanza, y mencionan que los estudiantes con mayores puntajes en las pruebas nacionales de admisión usualmente se concentran en las universidades ubicadas en la capital, por lo que el puntaje de corte nacional pone en desventaja a sus estudiantes y no reconoce el contexto regional, como lo describe uno de los participantes:

P: No existe una compresión de la población, de las localidades... Cualquier región en Chile tiene ciertas características que tienen que estar incluidas dentro del diagnóstico educativo... 550 puntos es el punto de la PSU, es una medida estándar que habla de una incomprensión y desconocimiento del territorio también, a eso me refiero; para mí, todas las políticas deben ser territorializadas, entendiéndose de la complejidad de donde se interviene educativamente (Director Pedagogía Media en Lenguaje, U5).

Consideran que la política no responde al perfil de sus estudiantes y su contexto; es decir, el contexto situado de estos directivos influye en sus críticas a la política (Ball et al., 2012). Los participantes reconocen que la política permite vías de admisión inclusivas; sin embargo, mencionan que no pueden admitir a todos sus estudiantes mediante estas vías, y reportan una baja en sus matrículas posterior a la aprobación de la política.

Estos programas han implementado cambios en sus currículums, alineándolos con los estándares nacionales y la prueba nacional diagnóstica del Ministerio de Educación. A diferencia del grupo anterior, mencionan que han desarrollado estos cambios sin dejar de lado su sello institucional, lo que se ejemplifica a continuación:

P: ... Vamos a tener que, desde las universidades, diseñar las formas de responder a estas expectativas o a este estándar o esta cosa externa, considerando nuestras propias características, y en eso estamos... Vamos buscando estrategias, formas, tratando de sistematizar, innovando en algunas otras cuestiones, como lo que yo te explicaba de las prácticas (Director Educación Parvularia, U1).

Asimismo, comentan que se encuentran en una tensión constante por mantener los énfasis de su formación, debido al gran número de estándares nacionales para la formación, y la presión de la evaluación externa a través de las pruebas diagnósticas y la acreditación. 


\section{Adaptación para sobrevivir al mercado}

Identificamos una cuarta tendencia entre ocho directores de programas de pedagogía de dos universidades privadas (2 y 9) con media o baja acreditación. Estos programas cuentan con varias sedes en diferentes regiones del país. Para los participantes, la política nacional responde a la alta heterogeneidad en la calidad y baja selectividad de los programas de pedagogía. Señalan que la política concibe a los profesores como personas capaces de reducir la brecha de aprendizaje, responder a la diversidad y trabajar en contextos de desventaja. A su juicio, la política promueve una formación docente alineada con las regulaciones y estándares nacionales, estableciendo requerimientos mínimos como vía para el mejoramiento y la autorregulación, como se ejemplifica a continuación:

P: Yo creo que viene a regularlo, yo creo que hoy día cualquier entidad que quiera convertirse en formadora de formadores tiene que cumplir un estándar mínimo, tiene que seleccionar a sus estudiantes y tiene que cumplir con ciertas condiciones mínimas, y sobre esa tiene que formar y poner sus sellos. Yo creo que esta ley ayuda a que la cancha se nivele... Claramente es un avance, es indiscutible (Director Pedagogía en Matemática, U2).

P: Nos obligan a mejorar el proceso de aprendizaje..., porque claro, debemos trabajar en razón a los estándares. O sea, que un profesor sepa enseñar, que un profesor sepa de su propia disciplina, que el profesor sepa de políticas y de normativas, que sepa comunicar, que sea reflexivo... Eso es un beneficio, porque nos ayuda a nosotros [a] tener una línea clara donde apuntar el aprendizaje. La dificultad es que hemos tenido que ajustar nuestros programas (Director Programa Pedagogía Básica, U9).

De manera similar a los participantes del grupo uno, los directores expresan una visión positiva respecto de la política, enfatizando su potencialidad para el mejoramiento de la formación docente y la equidad del sistema educativo.

Estos participantes reportan mayores cambios curriculares. Indican que sus programas se han adaptado a las demandas del SDPD, transitando de un foco en el conocimiento teórico y disciplinar, a la articulación teoría y práctica. Este cambio los llevó a crear iniciativas académicas para nivelar las competencias de entrada de los estudiantes. Explicitan que además de los cambios requeridos por la política, cuentan con cursos especiales para reforzar contenidos específicos antes de la evaluación nacional diagnóstica. A diferencia de los participantes del grupo dos y tres, estos directivos no critican ni muestran tensión ante los cambios. La cultura profesional de sus instituciones puede relacionarse con esta respuesta (Ball et al., 2012). En estos programas existían mecanismos de monitoreo interno previos a la política, y un currículum centralizado para asegurar una formación similar independiente de la sede y región. Esto puede relacionarse con la ausencia de críticas ante una política nacional que establece orientaciones curriculares centralizadas.

Estos participantes son también los que señalan más cambios en el perfil de sus estudiantes de pedagogía como resultado de la aprobación del SDPD. El promedio de admisión en la PSU se ha incrementado y el número de estudiantes de escuelas subvencionadas, también. Expresan preocupación por las consecuencias del aumento de selectividad en la matrícula de estudiantes de contextos en desventaja y para la sustentabilidad económica de sus programas. El incremento gradual de puntajes de selección a nivel nacional para estudiantes de pedagogía del SDPD hace dudar a los entrevistados sobre la competitividad de sus programas dentro de la universidad y su capacidad de matricular suficientes estudiantes para asegurar la rentabilidad. El contexto externo (Ball et al., 2012), la presión desde la política nacional por la acreditación, y la presión institucional por demostrar viabilidad económica es relevante para entender los cambios realizados en sus programas. 


\section{Discusión}

Este estudio contribuye a entender los factores que median la interpretación y respuesta (traducción) de las direcciones de programas de pedagogía a una política nacional que incrementa la estandarización y la rendición de cuentas. Con base en el análisis, identificamos dos dimensiones contextuales que influyen transversalmente en la puesta en práctica de la política en todos los grupos: la cultura profesional, y el contexto externo o las presiones externas (Ball et al., 2012). Adicionalmente, el contexto material o los recursos relacionados con la puesta en práctica de la política son aspectos relevantes para entender las respuestas de los grupos uno y dos, que han contado con recursos para hacer cambios, o cuentan con recursos limitados, respectivamente. Una cuarta dimensión, el contexto situado, es enfatizada solo por los participantes cuyos programas están ubicados fuera de la zona central del país.

Las interpretaciones y respuestas de las direcciones de los programas de pedagogía, cruzadas por las dimensiones contextuales, pueden entenderse en función de tres temáticas centrales: profesionalismo, justicia, y relaciones de poder.

El análisis muestra cómo las nociones de profesionalismo de los participantes se tensionan o confirman ante una política que aumenta la rendición de cuentas. Evetts (2009) señala que las políticas de rendición de cuentas promueven un profesionalismo organizacional, centrado en el control externo sobre resultados, la toma de decisiones jerárquica, la estandarización de los procesos y una autoridad basada en criterios legales o racionales, entre otras características. Sin embargo, Hardy \& Melville (2019) muestran cómo las nociones de profesionalismo de las políticas se reinterpretan y traducen en los contextos locales, dependiendo de las culturas profesionales docentes. En el contexto de la formación docente, este estudio concluye que la cultura profesional de los directores de programa tiene una fuerte influencia en sus interpretaciones y respuestas a la política. Los participantes del grupo uno y cuatro no expresan grandes discrepancias, desacuerdos o críticas con la política, valorando un mayor control nacional de la calidad de la formación. De manera coincidente, en sus instituciones existían mecanismos instalados de monitoreo interno sobre los programas de pedagogía. Los directores del segundo y tercer grupo, en cambio, criticaron los supuestos de la rendición de cuentas del SDPD, señalando que se asocian a prácticas gerenciales y jerárquicas, que contrastan con las relaciones profesionales de sus programas. Estos directores evidencian nociones de profesionalismo ocupacional (Evetts, 2009), en las que una buena formación docente es asociada a decisiones locales y colegiadas, la confianza y el juicio profesional sobre el trabajo. Las culturas profesionales, basadas en diferentes nociones de profesionalización, permiten comprender la aceptación o críticas a la política, y el alineamiento con o resistencia a ella.

Diversos estudios ahondan en prácticas de resistencia al analizar las implicancias de la instalación de las políticas en aspectos curriculares, pedagógicos y políticos (Henning, Dover, Dotson, \& Agarwal-Rangnath, 2018). Las críticas y respuestas a la política nacional de los directores de programa del segundo y tercer grupo muestran matices, que pueden ser categorizados bajo distintas nociones de resistencia. En el segundo grupo, las direcciones de programa critican fuertemente la política y enfatizan su compromiso con la educación pública; sin embargo, reconocen haber realizado cambios en su currículum para asegurarse de cubrir los contenidos declarados en los estándares nacionales y evaluados por la prueba nacional diagnóstica. Esta forma de resistencia o subversión encubierta (Henning et al., 2018) estaría movilizada por la tensión entre los compromisos éticos institucionales, que a su juicio no están presentes en la política, y a su calidad de funcionarios públicos, cuyo llamado es a alinearse con las directrices nacionales. En cambio, las direcciones de programa del tercer grupo, aun cuando reconocen cambios en el currículum basado en las demandas de la política, plantean una resistencia manifiesta (Henning et al., 2018). Esta resistencia se expresa no solo en el discurso, sino también en decisiones curriculares que buscan mantener las competencias del perfil de egreso y cursos asociados al sello institucional (religioso, local, o regional), a pesar de la constante presión por las prioridades que establece la política (estándares y contenidos de prueba diagnóstica). Así, la resistencia de los participantes a las políticas puede ser interpretada como una forma de salvaguardar su identidad profesional (Bolívar et al., 2005). 
Las nociones de justicia social que subyacen a los discursos de las direcciones de programa también permiten entender las diferentes interpretaciones y respuestas a la política. Los participantes de programas ubicados en regiones alejadas de la zona central (grupo 2 y 3 ) consideran que las evaluaciones externas (prueba diagnóstica nacional y acreditación) desconocen los aspectos contextuales de sus programas, lo que ha sido reportado en estudios internacionales sobre políticas de rendición de cuentas en formación docente (Bartlett et al, 2017; Reagan et al., 2016). El aumento de la selectividad de los estudiantes de pedagogía es otra de las tensiones señaladas por los participantes, debido a las dificultades para alcanzar los puntajes en la PSU exigidos en sus regiones. Estas tensiones se asocian a las nociones de justicia que subyacen a las críticas de los participantes, y que se centran en la ausencia de justicia distributiva en la política (Fraser \& Honneth, 2003); a su juicio, la política dificulta el acceso igualitario de estudiantes de regiones a programas de formación docente. Las medidas de incremento en la selectividad son consideradas como una amenaza al proyecto institucional, que enfatiza el servicio, acompańamiento y apoyo a las necesidades de sus estudiantes pese a sus bajos puntajes de ingreso. Por el contrario, la valoración positiva de la política por las direcciones de programa del grupo 1 y 4 pueden asociarse con la comprensión de la justicia distributiva como la posibilidad de la política de promover una docencia de calidad para todos los estudiantes de aula, avanzando en igualdad de oportunidades. Por otra parte, las críticas de las direcciones de programa de los grupos 2 y 3 a la política se relacionan con las dificultades que interpretan para alcanzar justicia de reconocimiento (Fraser \& Honneth, 2003). Mencionan que la política no considera el contexto y las necesidades locales a las que responden sus sellos, dificultando la mantención de un currículum que responda a la cultura de sus estudiantes. Estos esfuerzos se relacionan con dilemas éticos de la profesión docente y su responsabilización sobre la educación promovida (Escudero, 2011).

Otra temática relevante para entender las diferencias entre los participantes se relaciona con las relaciones de poder frente a las presiones externas (contexto externo) (Ball et al., 2012). De manera similar a los efectos perniciosos de las políticas de rendición de cuentas reportados en el sistema escolar (Stobart, 2008), el cuarto grupo de directores señala cambios en el currículum para priorizar los contenidos promovidos por la política nacional y el repaso de contenidos antes de la prueba nacional diagnóstica. El incremento de la selectividad y los requerimientos de acreditación presentan grandes desafíos para la sustentabilidad de sus programas y su posibilidad de sobrevivir con las nuevas reglas, dado que contaban con alta matrícula pero baja selectividad, y baja o media acreditación antes de la aprobación del SDPD. La percepción de escaso poder para cuestionar los cambios y mayor presión por realizarlos puede ayudar a entender por qué este grupo reporta más ajustes curriculares. El primer grupo, compuesto por directores de programas altamente selectivos, en universidades con alta acreditación, señala contar con mayores recursos externos para realizar innovaciones curriculares, y reportan mayor participación en la construcción de la política. Su mayor influencia sobre las decisiones de política les ha permitido anticiparse a los requerimientos de cambios u orientaciones, permitiendo entender por qué señalan escasos ajustes curriculares ante el SDPD. La producción de políticas es un proceso de disputa en el que las instituciones que se posicionan mejor serán capaces de incidir en un sistema educativo del cual comparten visiones e intereses (Levinson, Sutton, \& Winstead, 2009). En contraste, los directores de programas regionales señalan poca participación en la construcción de la política, y asumen una postura crítica hacia ella.

Estos hallazgos contribuyen a entender los acuerdos y tensiones de los directores de programa respecto de una política nacional que busca aumentar la selectividad y la rendición de cuentas de los programas de pedagogía. Específicamente, permiten comprender cómo los requerimientos de la política pueden tensionar las concepciones de profesionalización de los formadores docentes y sus propuestas formativas, interpelando su identidad profesional, cómo las consecuencias de estas políticas pueden ir en contra de prácticas que promueven la justicia distributiva o de reconocimiento en los programas, y la importancia de democratizar la participación y el poder de quienes ponen en práctica las políticas. El estudio contribuye, además, a expandir la escasa literatura sobre la puesta en práctica de políticas de rendición de cuentas en el campo de la formación docente, contexto en que se han 
incrementado. Nuestros hallazgos se basan en entrevistas con directores de programa, por lo que es necesario realizar investigación que incluya más fuentes de información y mayor variedad de actores en formación docente para profundizar y complejizar estos resultados desde los contextos locales.

El artículo original fue recibido el 20 de diciembre de 2019

El artículo revisado fue recibido el 21 de mayo de 2020

El artículo fue aceptado el 18 de junio de 2020

\section{Referencias}

Anderson, G. \& Cohen, M. (2018). The New Democratic Professional in Education: Confronting markets metrics and managerialism. Nueva York, NY: Teachers College Press.

Ball, S. (1993). What is policy? Texts, trajectories and toolboxes. Discourse: Studies in the Cultural Politics of Education, 13(2), 10-17. https://doi.org/10.1080/0159630930130203

Ball, S. (2001). Diretrizes Políticas Globais e Relaçôes Políticas Locais em Educação. Currículo sem Fronteiras, 1(2), 99-116. Recuperado de http://www.curriculosemfronteiras.org/volliss2articles/ball.htm

Ball, S., Maguire, M., \& Braun, A. (2012). How schools do policy. Policy enactment in secondary schools. Nueva York, NY: Routledge.

Ball, S., Maguire, M., Braun, A., \& Hoskins, K. (2011). Policy subjects and policy actors in schools: Some necessary but insufficient analyses. Discourse: Studies in the Cultural Politics of Education, 32(4), 611-624. https://doi.org/10.1080/01596306.2011.601564

Bartlett, M., Otis-Wilborn, A., \& Peters, L. (2017). Bending or breaking: Appropriating edTPA policy in special education teacher education. Teacher Education and Special Education, 40(4), 287-298. https://doi.org/10.1177/0888406417720188

Biblioteca del Congreso Nacional (BCN). (2016). Historia de la Ley 20.903: Crea el sistema de desarrollo profesional docente y modifica otras normas. Recuperado de https://www.bcn.cl/historiadelaley/nc/historia-de-la-ley/4890/

Biblioteca del Congreso Nacional (BCN). (2018). Nuestro pais: Ubicación y caracteristicas. Recuperado de https://www.bcn.cl/siit/nuestropais_14-7-2015

Bolívar, A., Gallego, M. J., León, M. J., y Pérez, P. (2005). Políticas educativas de reforma e identidades profesionales: El caso de la educación secundaria en España. Education Policy Analysis Archives, 13, 1-51. https://doi.org/10.14507/epaa.v13n45.2005

Bowe, R., Ball, S., \& Gold, A. (1992). Reforming education and changing schools: Case studies in policy sociology. Londres, Reino Unido: Routledge.

Butin, D. (2005). Is anyone listening? Educational policy perspectives on the social foundations of education. Educational studies, 38(3), 286-297. https://doi.org/10.1207/s15326993es3803_8

Cáceres, P. (2003). Análisis cualitativo de contenido: Una alternativa metodológica alcanzable. Psicoperspectivas, 2(1), 53-82. Recuperado de https://www.psicoperspectivas.cl/index.php/psicoperspectivas/article/view/3

Campbell, J. L., Quincy, C., Osserman, J., \& Pedersen, O. K. (2013). Coding in-depth semistructured interviews: Problems of unitization and intercoder reliability and agreement. Sociological Methods \& Research, 42(3), 294-320. https://doi.org/10.1177/0049124113500475

Centro de Perfeccionamiento, Experimentación e Investigación Pedagógica (CPEIP). (2017). Orientaciones Sistema de Desarrollo Profesional Docente. Santiago, Chile: División Jurídica Ministerio de Educación.

Cochran-Smith, M., Cummings Carney, M., Stringer Keefe, E., Burton, S., Chang, W.C., Fernández, M.B., Miller, A.F., Sánchez, J. G., \& Baker, M. (2018). Reclaiming Accountability in Teacher Education. New York, NY: Teachers College Press.

Creswell, J. M. (2009). Research design: Qualitative, quantitative, and mixed methods approaches. Thousand Oaks, CA: SAGE. 
Darling-Hammond, L. \& Lieberman, A. (2012). Teacher education around the World: Changing policies and practices. Nueva York, NY: Routledge.

Diem, S., Young, M., Welton, A., Cumings Mansfield, K., \& Lee, P. (2014). The intellectual landscape of critical policy analysis. International Journal of Qualitative Studies in Education, 27(9), 1068-1090. https://doi.org/10.1080/09518398.2014.916007

Elacqua, G., Hincapie, D., Vegas, E., y Alfonso, M. (2018). Profesión: Profesor en América Latina. ¿Por qué se perdió el prestigio docente y cómo recuperarlo? Washington, D.C.: Banco Interamericano de Desarrollo.

Escudero, J. M. (2011). Dilemas éticos de la profesión docente. Revista Participación Educativa, 16, 93-102. Recuperado de https://sede.educacion.gob.es/publiventa/participacion-educativa-n-16-revista-cuatrimestral-del-consejoescolar-del-estado-profesorado-y-calidad-de-la-educacion/educacion-profesores/14280

Evetts, J. (2009). New professionalism and new public management: Changes, continuities and consequences. Comparative Sociology, 8(2), 247-266. https://doi.org/10.1163/156913309X421655

Fernández, M.B. (2018). Framing teacher education: Conceptions of teaching, teacher education, and justice in Chilean national policies. Education Policy Analysis and Archives, 26(34), 1-37. https://doi.org/10.14507/epaa.26.2806

Fernández, M. B y Madrid, R. (2020). Profesionalización desde una perspectiva democrática: nudos críticos y propuestas para formación y desarrollo docente. En M. T. Corvera y G. Muñoz (Eds.), Horizontes y propuestas para transformar el sistema educativo chileno (pp. 206-233). Santiago, Chile: Biblioteca del Congreso Nacional.

Fraser, N. \& Honneth, A. (2003). Redistribution or recognition? A political-philosophical exchange. Nueva York, NY: Verso.

Fullan, M. (2013). Motion leadership in action. Thousand Oaks, CA: Corwin.

García-Huidobro, J. (2011). La Prueba Inicia: usos y abusos. Cuadernos de Educación, 29, 1-5. Recuperado de https://silo.tips/download/la-prueba-inicia-usos-y-abusos

Hargreaves, A. y Shirley, D. (2012). La cuarta vía: el prometedor futuro del cambio educativo. Barcelona, España: Ediciones Octaedro.

Hardy, I. \& Melville, W. (2019). Professional learning as policy enactment: The primacy of professionalism. Education Policy Analysis Archives, 27, 90. https://doi.org/10.14507/epaa.27.4401

Hasbun, T. \& Rudolph, A. (2016). Navigating the waters of accreditation: Best practices, challenges, and lessons learned from one institution. SAGE Open, 6(2), 1-10. https://doi.org/10.1177/2158244016656719

Henning, N., Dover, A. G., Dotson, E. K., \& Agarwal-Rangnath, R. (2018). Storying teacher education policy: Critical counternarratives of curricular, pedagogical, and activist responses to state-mandated teacher performance assessments. Education Policy Analysis Archives, 26, 26. https://doi.org/10.14507/epaa.26.2790

Hickok, E. W. (1998). Higher standards for teacher training. Policy Review, 91, 6-9.

Inzunza, J., Assaél, J., y Scherping, G. (2011). Formación docente inicial y en servicio en Chile: Tensiones de un modelo neoliberal. Revista Mexicana de Investigación Educativa (RMIE), 16(48), 267 -292. Recuperado de https://www.comie.org.mx/revista/v2018/rmie/index.php/nrmie/article/view/371

Ledwell, K. \& Oyler, C. (2016). Unstandardized responses to a "Standardized" test: The edTPA as gatekeeper and curriculumchangeagent.JournalofTeacherEducation, 67(2), 120-134. https://doi.org/10.1177/0022487115624739

Levinson, B. A., Sutton, M., \& Winstead, T. (2009). Education Policy as a Practice of Power: Theoretical Tools, Ethnographic Methods, Democratic Options. Educational Policy, 23(6), 767 - 795. https://doi.org/10.1177/0895904808320676

Ministerio de Educación (MINEDUC). (2018). Establece distribución de recursos, en virtud del literal A) de la fórmula de cálculo establecida por el artículo trigésimo octavo transitorio de la Ley N²1.091, entre las Instituciones de Educación Superior que indica, beneficiarias del financiamiento institucional para gratuidad en el año académico 2018. Decreto 203 del 8 de octubre 2018. Santiago. Recuperado de https://transparencia.uda.cl/ files/beca/Decreto\%20302\%202018\%20DISTRIB\%20RECURSOS\%20GRATUIDAD.pdf

Mitchell, D. \& Romero, L. (2010). The politics and practice of alternative teacher certification. Education Administration Quarterly, 46(3), 363-394. https://doi.org/10.1177/0013161X10375272

Montecinos, C. (2014). Análisis crítico de las medidas de presión propuestas para mejorar la formación inicial de docentes en Chile por el panel de expertos para una educación de calidad. Estudios Pedagógicos (Valdivia), 40(especial), 285-301. https://doi.org/10.4067/S0718-07052014000200017 
Muñoz, M. y Blanco, C. (2013). Una taxonomía de las universidades. Calidad en la Educación, (38), 181-213. https://doi.org/10.4067/S0718-45652013000100005

Parcerisa, L. y Verger, A. (2016). Rendición de cuentas y política educativa: Una revisión de la evidencia internacional y futuros retos para la investigación. Profesorado: Revista de Curriculum y Formación de Profesorado, 20(3), 15-51.

Peck, C. A., Gallucci, C., \& Sloan, T. (2010). Negotiating implementation of high-stakes performance assessment policies in teacher Education: From compliance to inquiry. Journal of Teacher Education, 61(5), 451-463. https://doi.org/10.1177/0022487109354520

Petrelli, L. (2014). Formación docente, estado y apropiación: notas sobre la implementación de un nuevo plan de estudio en la carrera docente. Propuesta Educativa, (42), 65-76. Recuperado de http://propuestaeducativa.flacso.org.ar/ wp-content/uploads/2019/12/42-art-petrelli.pdf

Reagan, E., Schram, T., Mc Curdy, K., Chang, T., \& Evans, C. (2016). Politics of policy: Assessing the implementation, impact, and evolution of the performance assessment for California teachers (PACT) and edTPA. Education Policy Analysis Archives, 24, 9. https://doi.org/10.14507/epaa.24.2176

Ruffinelli, A. (2016). Ley de desarrollo profesional docente en Chile: de la precarización sistémica de los logros, avances y desafíos pendientes para la profesionalización. Estudios Pedagógicos (Valdivia), 42(4), 261 -279. https://doi.org/10.4067/S0718-07052016000500015

Sotomayor, C. y Gysling, J. 2011). Estándares y regulación de calidad de la formación de profesores: discusión del caso chileno desde una perspectiva comparada. Calidad de la Educación, (35), 91 - 129. https://doi.org/10.4067/S0718-45652011000200004

Stobart, G. (2008). Testing times. The uses and abuses of assessment. Nueva York, NY: Routledge.

Stone, D. (2012). Policy paradox: The art of political decision making. Nueva York, NY: Norton.

Torres, R. y Zenteno, M. (2011). El sistema de educación superior: una mirada desde las instituciones y sus características. En M. Jiménez de la Jara \& F. Lagos (Eds.), Nueva geografía del sistema de educación superior y de los estudiantes (pp. 13-77). Santiago, Chile: Aequalis.

Verger, A. (2013). Políticas de mercado, Estado y universidad: hacia una conceptualización y explicación del fenómeno de la mercantilización de la educación superior. Revista de Educación, 360, 268-291. https://doi.org/10.4438/1988-592X-RE-2011-360-111

Wood, P. y Smith, J. (2018). Investigar en educación: Conceptos básicos y metodologías para desarrollar proyectos de investigación. Madrid, España: Narcea.

Zeichner, K. (2010). Competition, economic rationalization, increased surveillance, and attacks on diversity: Neoliberalism and the transformation of teacher education in the US. Teaching and Teacher Education, 26(8), 1544-1552. https://doi.org/10.1016/j.tate.2010.06.004 